\title{
Efficacy and Safety of Sitagliptin in Hispanic/Latino Patients with Type 2 Diabetes: A Pooled Analysis from Ten Randomized, Placebo-Controlled Phase 3 Clinical Trials
}

\author{
Annaswamy Raji (D) - Jianmin Long - Raymond L. H. Lam • \\ Edward A. O’Neill · Samuel S. Engel
}

Received: May 24, 2018 / Published online: June 23, 2018

(C) The Author(s) 2018

\section{ABSTRACT}

Introduction: To assess the efficacy and safety profile of the dipeptidyl-peptidase- 4 inhibitor sitagliptin in a population of self-identified Hispanic/Latino patients with type 2 diabetes.

Methods: Data were pooled from ten randomized, double-blind studies in which subjects were treated with sitagliptin $100 \mathrm{mg} /$ day (as mono- or combination therapy) or placebo, and used to evaluate the glycemic efficacy, safety, and tolerability of sitagliptin compared with placebo after 24 weeks of treatment.

Results: A total of 804 Hispanic/Latino patients were included in the analysis. Baseline characteristics in the treatment groups were similar (mean baseline HbA1c of approximately 8.5\%). The LS mean HbA1c changes from baseline were $-0.94 \%$ with sitagliptin and $-0.32 \%$ with placebo, and the between-group difference was $-0.62 \%, p<0.001$. After 24 weeks of treatment, $35 \%$ and $18 \%$ of subjects were at the HbA1c goal of $<7 \%$ in the sitagliptin and placebo groups, respectively. Body weight increased slightly in both treatment groups.

Enhanced digital features To view enhanced digital features for this article go to https://doi.org/10.6084/ m9.figshare.6477737.

A. Raji $(\bowtie) \cdot$ J. Long $\cdot$ R. L. H. Lam ·

E. A. O’Neill · S. S. Engel

Merck \& Co., Inc., Kenilworth, NJ, USA

e-mail: annaswamy.raji@merck.com
Incidences of adverse events of hypoglycemia were similar and low (1.9\% and $1.4 \%$ for sitagliptin and placebo, respectively) in both groups in studies in which insulin or sulfonylurea were not used and were similar (9\% and $11 \%$ for sitagliptin and placebo, respectively) when all studies were included. Overall safety and tolerability of treatment with sitagliptin and placebo were similar. No clinically meaningful differences between the safety profile of sitagliptin in the Hispanic/Latino population analyzed here and broader populations previously evaluated were observed.

Conclusion: In this pooled analysis of sitagliptin therapy vs placebo in Hispanic/Latino patients, sitagliptin provided significant improvement in glycemic control and was generally well tolerated.

Funding: Merck \& Co., Inc., Kenilworth, NJ, USA.

Keywords: DPP-4 inhibitor; Ethnicity; HbA1c; Hispanic/Latino; Incretin therapy; Type 2 diabetes

\section{INTRODUCTION}

Although differences in response to drug treatment have been observed in racially distinct subgroups [1, 2] (potentially attributable to genetics, metabolism, diet, environmental exposure, and/or sociocultural issues [3]), in 
general, new treatments are neither designed for nor tested in specific racial or ethnic groups. Instead, clinical trials usually include people of diverse racial and ethnic backgrounds, with primary safety and efficacy analyses based on results in the racially and ethnically mixed group. In any trial, an approximation of the safety and efficacy of a treatment can be made on the basis of subgroup analyses, but such analyses often do not include a sufficient number of participants to provide statistically significant results and robust conclusions. However, when a treatment has been evaluated in a sufficiently large number of similar trials, it becomes possible to analyze pooled subgroup data.

The prevalence of diagnosed and undiagnosed type 2 diabetes (T2D) among adults of Hispanic ethnicity in the USA is estimated to be $16.4 \%$ [4]. The prevalence of diagnosed diabetes among adults is estimated to be $8.0 \%$ and growing in South and Central America [5] and $13.8 \%$ in Spain [6]. Despite this, a limited amount of information is available describing the effects of the antihyperglycemic medication sitagliptin in the Hispanic population. However, a subgroup of patients self-identifying as Hispanic or Latino ethnicity (hereafter referred to as Hispanic) participate in most clinical trials of sitagliptin. In the analysis reported here, data from ten phase 3, randomized, double-blind studies in which subjects were treated with sitagliptin or placebo were pooled to evaluate the glycemic efficacy, safety, and tolerability of sitagliptin compared with placebo in the selfidentified Hispanic population participating in these studies.

\section{METHODS}

\section{Data Sources}

Data for this analysis were taken from ten randomized, double-blind clinical studies ranging from 24 to 54 weeks duration; two were of sitagliptin monotherapy [7, 8], two of sitagliptin add-on to metformin $[9,10]$, and one each of sitagliptin add-on to pioglitazone [11], to metformin + rosiglitazone

[12], metformin + pioglitazone

[13], to insulin \pm metformin [14], to insulin \pm metformin or sulfonylurea [15], and to glimepiride or glimepiride + metformin [16]. In all but two studies $[10,12]$, the primary study duration ran through week 24 , during which period subjects were treated with sitagliptin $100 \mathrm{mg} /$ day or placebo as monotherapy or as add-on therapy. The primary endpoint for studies [10] and [12] was week 18 , but [10] ran through week 30 and [12] through week 54 . All procedures performed in the studies involving human participants were in accordance with the 1964 Declaration of Helsinki and its later amendments or comparable ethical standards. Informed consent was obtained from all individual participants in all of these studies.

\section{Endpoints}

All endpoints were analyzed at (or through) week 24 , the primary time point common to most studies. Efficacy endpoints were change from baseline in HbA1c at week 24 and percentage of patients at HbA1c goal $<7 \%$ at week 24. Safety endpoints were change from baseline in body weight at week 24 , incidence of adverse events (AEs) collected through week 24, and incidence of hypoglycemia AEs through week 24.

\section{Statistical Analyses}

The analysis population consisted of all randomized patients who took at least one dose of study medication. For change from baseline analysis, patients were required to have measurements both at baseline and post-baseline at or prior to the analysis endpoint. Missing data were imputed using the last-observation-carried-forward approach for efficacy endpoints but not for the safety endpoints. Data acquired after the initiation of rescue therapy were treated as missing to avoid the confounding influence of rescue therapy. Descriptive statistics were used to summarize demographic variables and baseline characteristics. An analysis of covariance model including a term for treatment and baseline value as a covariate was used 
for the assessment of continuous endpoints (HbA1c, fasting plasma glucose, body weight). The Miettinen and Nurminen method [17] was used to calculate the $95 \%$ confidence intervals (CI) and $p$ values for the between-group differences in percentage of patients at HbA1c goal and incidence rate of AE endpoints. Subgroup analysis was performed for subgroups defined by sex, age, body mass index (BMI), and baseline HbA1c categories.

\section{RESULTS}

In the ten studies that provided data for this analysis, there were 804 Hispanic patients ( $n=439$ treated with sitagliptin, $n=365$ with placebo) whose data were appropriate for inclusion in the analysis. Baseline demographic and anthropometric characteristics were similar in the two treatment groups (Table 1). For comparison, data from the non-Hispanic patients in these studies $(n=1837$ treated with sitagliptin, $n=1617$ with placebo) were also pooled and analyzed. Baseline demographics of the non-Hispanic group were similar to the Hispanic groups (Table 1), except that the nonHispanic population was slightly older, with a smaller percentage of female patients and slightly higher body weight and BMI.

At week 24, in the Hispanic population the LS mean (95\% CI) changes from baseline in HbA1c were $-0.94 \%(-1.05,-0.83)$ with sitagliptin and $-0.32 \%(-0.44,-0.20)$ with placebo; the between-group difference was $-0.62 \%(-0.78,-0.46), p<0.001$ (Fig. 1). At week $24,34.7 \%$ of Hispanic patients treated with sitagliptin were at the HbA1c goal of $<7 \%$ compared with $17.8 \%$ of those treated with placebo (Fig. 2).

The between-group difference in the LS mean $(95 \% \mathrm{CI})$ change from baseline in HbA1c in the sitagliptin treatment group of the Hispanic population was similar to that of the nonHispanic population $[-0.68 \%(-0.75,-0.62)]$ (Fig. 1). In addition, the percentage of patients at HbA1c goal of $<7 \%$ in the Hispanic population was similar to that in the non-Hispanic population (31.7\%) (Fig. 2).
The efficacy of sitagliptin was also evaluated in subgroups of the Hispanic population defined by age, sex, baseline BMI, or baseline HbA1c. In all subgroups the effect of sitagliptin on change from baseline HbA1c compared with placebo was consistent with that observed in the entire Hispanic population (Fig. 3).

At week 24, the LS mean body weight increased slightly in both treatment groups of the Hispanic population, resulting in no significant change associated with active treatment (Table 2). In subgroups of patients analyzed by BMI, similar results were observed (Table 2).

There were no meaningful differences between treatment groups in incidences of overall AEs, drug-related AEs, serious AEs, serious drug-related AEs or deaths in the Hispanic population (Table 3). The incidence of hypoglycemia was low in both treatment groups of Hispanic patients who were not simultaneously treated with a sulfonylurea or insulin (Table 3). In both the sitagliptin- and placebo-treated patient groups, the incidence of hypoglycemia was higher in the populations of patients who were being treated with a sulfonylurea or insulin (Table 3). There were no events of severe hypoglycemia in either treatment group.

\section{DISCUSSION}

There is paucity of data evaluating the safety and efficacy of antihyperglycemic agents in patients with T2D who classify themselves as Hispanic. Here, data were pooled from patients self-identifying as Hispanic who participated in any of ten phase 3 clinical studies which compared the efficacy and safety of sitagliptin with placebo. The results of this analysis demonstrate that in Hispanic patients sitagliptin improves glycemic control and is generally well tolerated compared with placebo.

In the analyses of efficacy by subgroups of Hispanic patients, the effect of sitagliptin treatment on change from baseline in the LS mean HbA1c was consistent with its effect in the overall Hispanic population, and with its effect in the non-Hispanic population. 
Table 1 Baseline demographic, anthropometric, and disease characteristics of trial treatment groups safety population

\begin{tabular}{llllll}
\hline & \multicolumn{3}{l}{ Hispanic } & & Non-Hispanic \\
\cline { 2 - 3 } \cline { 5 - 5 } Sitagliptin, $\boldsymbol{n}=\mathbf{4 3 9}$ & Placebo, $\boldsymbol{n}=\mathbf{3 6 5}$ & & Sitagliptin, $\boldsymbol{n}=\mathbf{1 8 3 7}$ & Placebo, $\boldsymbol{n}=\mathbf{1 6 1 7}$ \\
\hline Age, years & $54.5 \pm 10.2$ & $55.1 \pm 10.3$ & $57.1 \pm 10.1$ & $57.7 \pm 10.2$ \\
Female, $n$ (\%) & $248(56.5)$ & $199(54.5)$ & $825(44.9)$ & $717(44.3)$ \\
Weight, kg & $79.1 \pm 16.6$ & $79.5 \pm 17.2$ & $87.6 \pm 18.9$ & $88.1 \pm 19.5$ \\
BMI, kg/m & $30.4 \pm 5.1$ & $30.5 \pm 5.0$ & $31.2 \pm 5.7$ & $31.3 \pm 5.8$ \\
HbA1c, \% & $8.5 \pm 1.0$ & $8.6 \pm 1.1$ & $8.4 \pm 1.0$ & $8.4 \pm 0.9$ \\
FPG, mg/dL & $171.7 \pm 45.8$ & $168.6 \pm 40.9$ & $176.9 \pm 44.0$ & $178.7 \pm 46.8$ \\
Duration of T2D, years & $9.1 \pm 7.0$ & $9.6 \pm 6.7$ & $8.6 \pm 6.5$ & $9.0 \pm 6.8$ \\
Background AHA, $n(\%)$ & & & & \\
Glimepiride & $26(5.9)$ & $25(6.8)$ & $80(4.4)$ & $81(5.0)$ \\
Insulin & $136(31.0)$ & $123(33.7)$ & $516(28.1)$ & $526(32.5)$ \\
Metformin & $103(23.5)$ & $52(14.2)$ & $457(24.9)$ & $279(17.3)$ \\
TZD & $21(4.8)$ & $22(6.0)$ & $154(8.4)$ & $156(9.6)$ \\
Metformin + glimepiride & $13(3.0)$ & $7(1.9)$ & $103(5.6)$ & $106(6.6)$ \\
Metformin + TZD & $73(16.6)$ & $66(18.1)$ & $254(13.8)$ & $182(11.3)$ \\
No background AHA & $67(15.3)$ & $70(19.2)$ & $273(14.9)$ & $287(17.7)$ \\
\hline
\end{tabular}

Values are mean \pm standard deviation unless otherwise noted $B M I$ body mass index, $F P G$ fasting plasma glucose, $T 2 D$ type 2 diabetes mellitus, $A H A$ antihyperglycemic agent, $T Z D$ thiazolidinedione

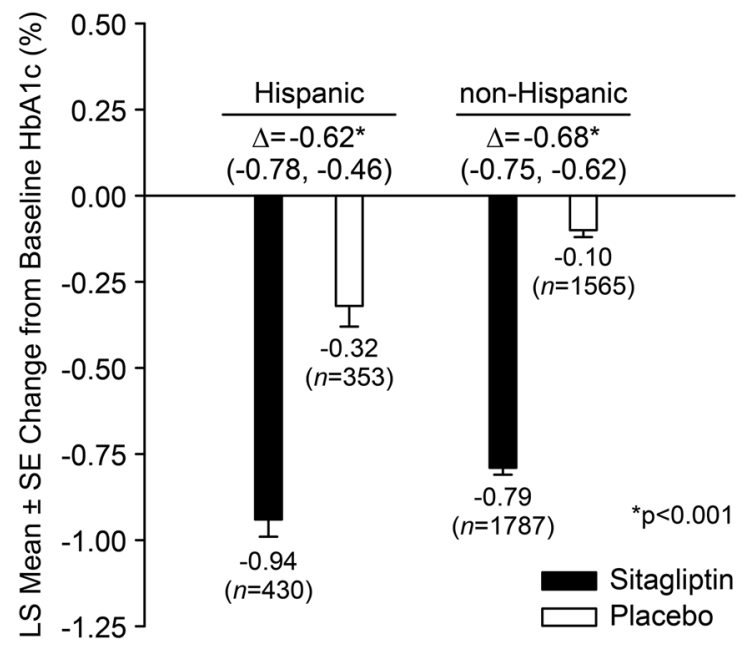

Fig. 1 Change from baseline in HbAlc (\%) at week 24

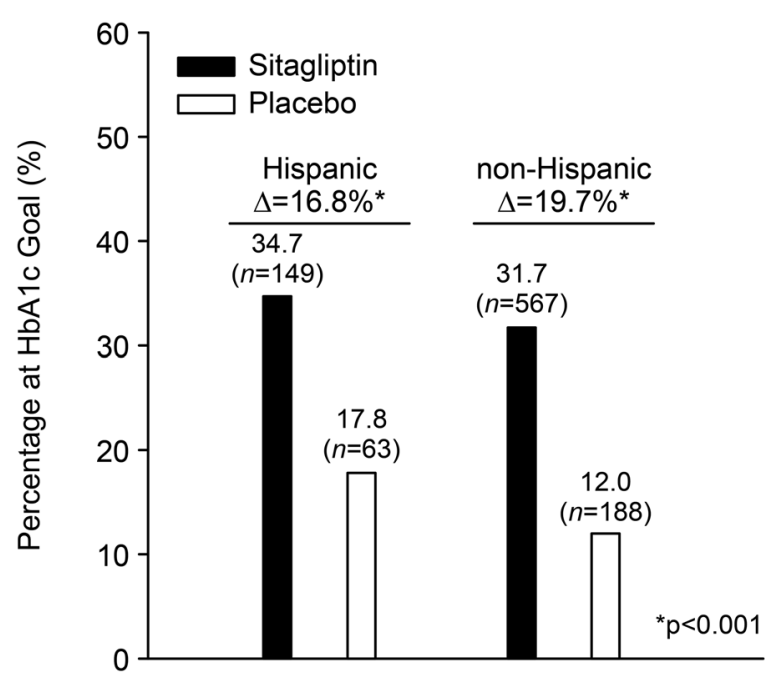

Fig. 2 Percentage of patients at HbAlc goal of $<7 \%$ at week 24 
(A)

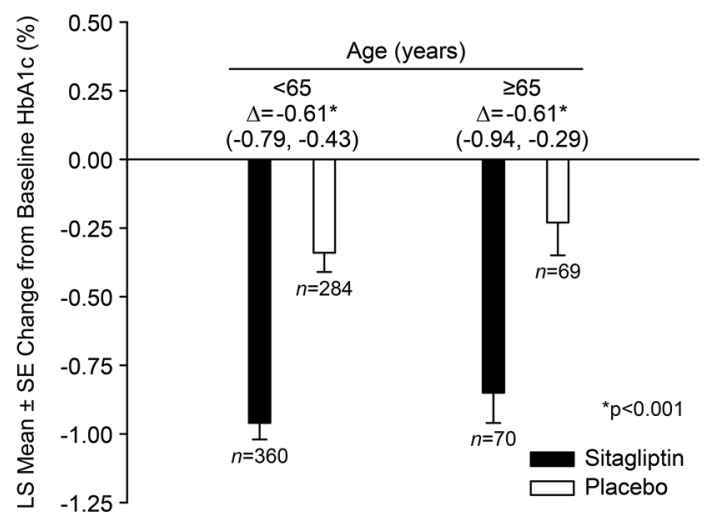

(B)

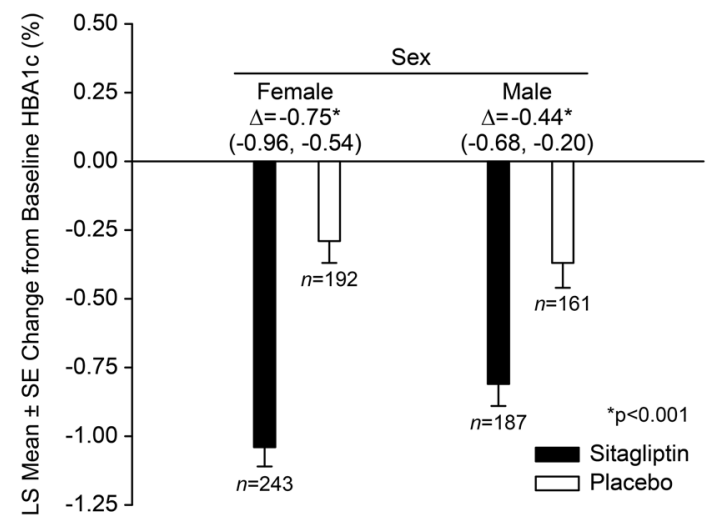

(C)

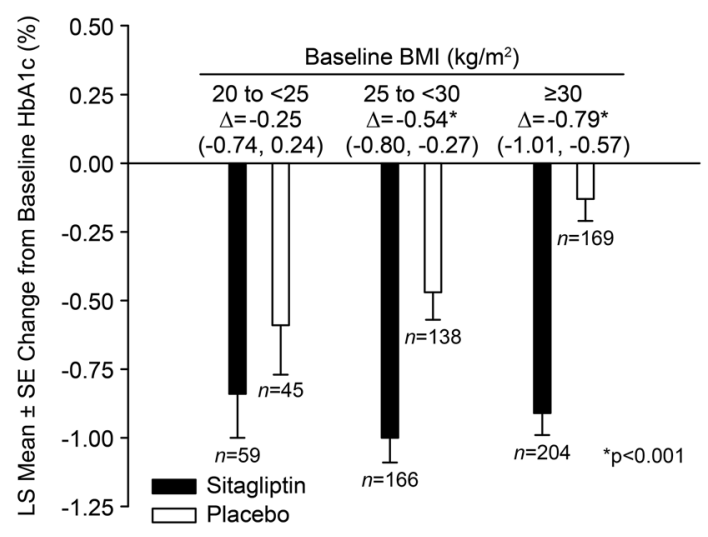

(D)

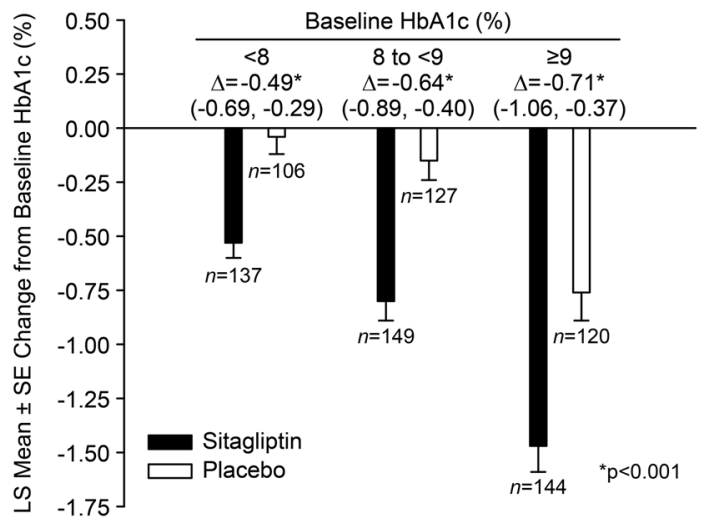

4 Fig. 3 Change from baseline in HbAlc at week 24 in Hispanic patient subgroups by a age, $\mathbf{b}$ sex, $\mathbf{c}$ baseline body mass index (BMI), $\mathbf{d}$ baseline HbAlc

The summary AE analysis for the Hispanic subgroup demonstrated no imbalance between the sitagliptin and placebo treatment groups. This is consistent with a previously published, extensive, and detailed safety analysis of pooled data from 25 studies with sitagliptin, which suggested no association of sitagliptin use with any AEs other than hypoglycemia (when used with SUs or insulin) [18].

Unlike AHAs such as insulin and sulfonylureas, across numerous clinical studies sitagliptin has consistently proven to be weight neutral [19]. Consistent with these observations, in the Hispanic population analyzed here, sitagliptin treatment for 24 weeks was weight neutral in the entire population, as well as in all subgroups evaluated by BMI.

Increased incidence of hypoglycemia is associated with the use of insulin and sulfonylureas [20]. Hypoglycemia is associated with increased morbidity and, in some studies, with increased mortality [21] and it is known to have a deleterious effect on patients' quality of life [22] and medication adherence [23]. Consistent with results obtained in ethnically diverse studies [18], in this analysis of Hispanic patients, the incidence of hypoglycemia associated with sitagliptin treatment alone was low and similar to treatment with placebo. In addition, while the incidence of hypoglycemia during treatment with sitagliptin in combination with an SU or insulin was higher than with sitagliptin alone, again it was similar to placebo treatment in combination with an SU or insulin.

The glycemic efficacy and safety profile of sitagliptin in Hispanic patients observed in the analysis reported here are consistent with those previously reported for the entire populations of patients who participated in the studies which provided the data for this analysis and with those observed in the non-Hispanic cohort in the same trials. The results are also consistent with a similar analysis of Hispanic patients 
Table 2 Change from baseline in body weight $(\mathrm{kg})$ in the Hispanic population

\begin{tabular}{|c|c|c|}
\hline & Sitagliptin & Placebo \\
\hline \multicolumn{3}{|l|}{ Entire population } \\
\hline Overall population & $N=369$ & $N=283$ \\
\hline Baseline & $78.6 \pm 16.8$ & $77.6 \pm 15.8$ \\
\hline Week 24 & $79.3 \pm 16.7$ & $78.3 \pm 16.2$ \\
\hline Change from baseline ${ }^{a}$ & $0.62(0.34,0.91)$ & $0.68(0.36,1.01)$ \\
\hline Change vs. placebo ${ }^{b}$ & $-0.06(-0.50,0.38)$ & - \\
\hline \multicolumn{3}{|c|}{ Subgroups by baseline BMI, $\mathrm{kg} / \mathrm{m}^{2}$} \\
\hline 20 to $<25$ & $n=50$ & $n=40$ \\
\hline Baseline & $60.9 \pm 7.7$ & $58.5 \pm 6.7$ \\
\hline Week 24 & $62.0 \pm 8.0$ & $59.1 \pm 6.6$ \\
\hline Change from baseline $\mathrm{a}^{\mathrm{a}}$ & $1.06(0.51,1.61)$ & $0.65(0.03,1.27)$ \\
\hline Change vs. placebo ${ }^{\mathrm{b}}$ & $0.41(-0.43,1.24)$ & - \\
\hline 25 to $<30$ & $n=144$ & $n=117$ \\
\hline Baseline & $71.8 \pm 9.3$ & $71.6 \pm 8.6$ \\
\hline Week 24 & $72.7 \pm 9.8$ & $72.2 \pm 9.0$ \\
\hline Change from baseline ${ }^{a}$ & $0.84(0.41,1.27)$ & $0.69(0.22,1.17)$ \\
\hline Change vs. placebo ${ }^{\mathrm{b}}$ & $0.15(-0.49,0.79)$ & - \\
\hline$\geq 30$ & $n=175$ & $n=125$ \\
\hline Baseline & $89.3 \pm 16.2$ & $89.5 \pm 13.8$ \\
\hline Week 24 & $89.6 \pm 16.3$ & $90.2 \pm 14.5$ \\
\hline Change from baseline ${ }^{a}$ & $0.32(-0.16,0.79)$ & $0.69(0.13,1.26)$ \\
\hline Change vs. placebo ${ }^{b}$ & $-0.38(-1.11,0.36)$ & - \\
\hline
\end{tabular}

Values are mean \pm standard deviation unless otherwise noted

a Least squares (LS) mean (95\% CI)

b Difference in LS means (95\% CI); all $p>0.05$

using data pooled from studies of linagliptin, another dipeptidyl-peptidase-4 inhibitor [24].

There are several limitations to the analysis presented here. First, it is a pooled analysis of data from studies with varying designs and background medications. Second, the sizes of the subgroup populations may not be sufficiently powered to discern small differences among subgroups. Third, patients self-classifying as Hispanic or Latino do not comprise a population sharing a unique set of genetic, cultural, or environmental influences and thus the results of this analysis may not be relevant to every individual self-identifying as Hispanic or Latino.

\section{CONCLUSIONS}

In this analysis of data pooled from ten randomized, placebo-controlled clinical studies, sitagliptin treatment of self-identifying 
Table 3 Adverse events summary

\begin{tabular}{|c|c|c|c|}
\hline Subjects, $n$ (\%) & Sitagliptin, $N=439$ & Placebo, $N=365$ & Difference $^{a}$ \\
\hline \multicolumn{4}{|l|}{ With one or more } \\
\hline AEs & $262(59.7)$ & $210(57.5)$ & $2.1(-4.7,9.0)$ \\
\hline Drug-related ${ }^{\mathrm{b}}$ AEs & $38(8.7)$ & $28(7.7)$ & $1.0(-2.9,4.8)$ \\
\hline Serious AEs & $13(3.0)$ & $12(3.3)$ & $-0.3(-3.0,2.2)$ \\
\hline Serious drug-related ${ }^{\mathrm{b}} \mathrm{AEs}$ & $1(0.2)$ & $1(0.3)$ & $-0.0(-1.3,1.0)$ \\
\hline Who died & $0(0)$ & $1(0.3)$ & $-0.3(-1.5,0.6)$ \\
\hline \multicolumn{4}{|l|}{ Who discontinued because of } \\
\hline An $\mathrm{AE}$ & $7(1.6)$ & $6(1.6)$ & $-0.0(-2.1,1.8)$ \\
\hline A drug-related ${ }^{\mathrm{b}} \mathrm{AE}$ & $3(0.7)$ & $2(0.5)$ & $0.1(-1.4,1.5)$ \\
\hline A serious $\mathrm{AE}$ & $1(0.2)$ & $3(0.8)$ & $-0.6(-2.2,0.5)$ \\
\hline A serious drug-related ${ }^{\mathrm{b}} \mathrm{AE}$ & $1(0.2)$ & $1(0.3)$ & $-0.0(-1.3,1.0)$ \\
\hline With AEs of hypoglycemia & $38(8.7)$ & $39(10.7)$ & $-2.0(-6.3,2.1)$ \\
\hline On SU or insulin & $33 / 175(18.9)$ & $36 / 155(23.2)$ & $-4.4(-13.3,4.4)$ \\
\hline Not on SU or insulin & $5 / 264(1.9)$ & $3 / 210(1.4)$ & $0.5(-2.4,3.1)$ \\
\hline Severe ${ }^{c}$ & $0(0)$ & $0(0)$ & - \\
\hline
\end{tabular}

$S U$ sulfonylurea

a Difference in \%, sitagliptin vs. placebo; estimate (95\% CI)

b Assessed by the investigator as related to study drug

c Severe hypoglycemia: episode that required assistance, either medical or non-medical. Episodes with a markedly depressed level of consciousness, a loss of consciousness, or seizure would be classified as having required medical assistance, whether or not medical assistance was obtained

Hispanic patients with T2D provided clinically meaningful improvement in glycemic control. In addition, the safety profile of sitagliptin in this population of patients was similar to that previously observed in ethnically mixed patient populations.

\section{ACKNOWLEDGEMENTS}

We thank the participants of the ten clinical trials. Written permission was obtained from all authors and all acknowledged contributors associated with this manuscript.

Funding. Funding for this study and payment for article processing charges were provided by Merck \& Co., Inc., Kenilworth, NJ,
USA. All authors had full access to all of the data in this study and take complete responsibility for the integrity of the data and accuracy of the data analysis.

Editorial Assistance. Editorial assistance was provided by Jennifer Rotonda $\mathrm{PhD}$ and Michele McColgan BA of Merck \& Co., Inc., Kenilworth, NJ, USA and was funded by Merck \& Co., Inc., Kenilworth, NJ, USA.

Authorship. All named authors meet the International Committee of Medical Journal Editors (ICMJE) criteria for authorship for this article, take responsibility for the integrity of the work as a whole, and have given their approval for this version to be published. 
Authorship Contributions. Annaswamy Raji and Samuel S. Engel conceived, designed, and/ or planned the study. Annaswamy Raji and Jianmin Long analyzed the data. Annaswamy Raji, Jianmin Long, Raymond L.H. Lam, Edward A. O'Neill, and Samuel S. Engel interpreted the results. Annaswamy Raji and Edward A. O'Neill drafted the manuscript. Annaswamy Raji, Jianmin Long, Raymond L.H. Lam, and Samuel S. Engel critically reviewed and/or revised the manuscript for important intellectual content.

Disclosures. Annaswamy Raji is a current employee of Merck Sharp \& Dohme Corp., a subsidiary of Merck \& Co. Inc., Kenilworth, NJ, USA, and may own company stock and/or hold stock options in the company. Jianmin Long is a former employee of Merck Sharp \& Dohme Corp., a subsidiary of Merck \& Co. Inc., Kenilworth, NJ, USA, and may own company stock and/or hold stock options in the company. Raymond L. H. Lam is a current employee of Merck Sharp \& Dohme Corp., a subsidiary of Merck \& Co. Inc., Kenilworth, NJ, USA, and may own company stock and/or hold stock options in the company. Edward A. O'Neill is a current employee of Merck Sharp \& Dohme Corp., a subsidiary of Merck \& Co. Inc., Kenilworth, NJ, USA, and may own company stock and/or hold stock options in the company. Samuel S. Engel is a current employee of Merck Sharp \& Dohme Corp., a subsidiary of Merck \& Co. Inc., Kenilworth, NJ, USA, and may own company stock and/or hold stock options in the company.

Compliance with Ethics Guidelines. All procedures performed in the studies involving human participants were in accordance with the 1964 Declaration of Helsinki and its later amendments or comparable ethical standards. Informed consent was obtained from all individual participants in all of these studies.

Data Availability. Merck \& Co., Inc.'s data sharing policy, including restrictions, is available at http://engagezone.merck.com/ds_docu mentation.php. Requests for access to the study data can be submitted through the EngageZone site or via email to dataaccess@merck.com.
Open Access. This article is distributed under the terms of the Creative Commons Attribution-NonCommercial 4.0 International License (http://creativecommons.org/licenses/ by-nc/4.0/), which permits any noncommercial use, distribution, and reproduction in any medium, provided you give appropriate credit to the original author(s) and the source, provide a link to the Creative Commons license, and indicate if changes were made.

\section{REFERENCES}

1. Carson P, Ziesche S, Johnson G, Cohn JN. Racial differences in response to therapy for heart failure: analysis of the vasodilator-heart failure trials. Vasodilator-Heart Failure Trial Study Group. J Card Fail. 1999;5:178-87.

2. Ramamoorthy A, Pacanowski MA, Bull J, Zhang L. Racial/ethnic differences in drug disposition and response: review of recently approved drugs. Clin Pharmacol Ther. 2015;97:263-73.

3. Huang SM, Temple R. Is this the drug or dose for you? Impact and consideration of ethnic factors in global drug development, regulatory review, and clinical practice. Clin Pharmacol Ther. 2008;84:287-94.

4. National Center for Chronic Disease Prevention and Health Promotion, Division of Diabetes Translation. National Diabetes Statistics Report, 2017; estimates of diabetes and its burden in the United States. 2017. https://www.cdc.gov/diabetes/pdfs/ data/statistics/national-diabetes-statistics-report. pdf. Accessed 18 Jun 2018.

5. Aschner P, Aguilar-Salinas C, Aguirre L, et al. Diabetes in South and Central America: an update. Diabetes Res Clin Pract. 2014;103:238-43.

6. Soriguer F, Goday A, Bosch-Comas A, et al. Prevalence of diabetes mellitus and impaired glucose regulation in Spain: the Di@bet.es Study. Diabetologia. 2012;55:88-93.

7. Aschner P, Kipnes MS, Lunceford JK, Sanchez M, Mickel C, Williams-Herman DE. Effect of the dipeptidyl peptidase-4 inhibitor sitagliptin as monotherapy on glycemic control in patients with type 2 diabetes. Diabetes Care. 2006;29:2632-7.

8. Barzilai N, Guo H, Mahoney EM, et al. Efficacy and tolerability of sitagliptin monotherapy in elderly patients with type 2 diabetes: a randomized, 
double-blind, placebo-controlled trial. Curr Med Res Opin. 2011;27:1049-58.

9. Charbonnel B, Karasik A, Liu J, Wu M, Meininger G. Efficacy and safety of the dipeptidyl peptidase- 4 inhibitor sitagliptin added to ongoing metformin therapy in patients with type 2 diabetes inadequately controlled with metformin alone. Diabetes Care. 2006;29:2638-43.

10. Raz I, Chen Y, Wu M, et al. Efficacy and safety of sitagliptin added to ongoing metformin therapy in patients with type 2 diabetes. Curr Med Res Opin. 2008;24:537-50.

11. Rosenstock J, Brazg R, Andryuk PJ, Lu K, Stein P. Efficacy and safety of the dipeptidyl peptidase- 4 inhibitor sitagliptin added to ongoing pioglitazone therapy in patients with type 2 diabetes: a 24-week, multicenter, randomized, double-blind, placebocontrolled, parallel-group study. Clin Ther. 2006;28:1556-68.

12. Dobs AS, Goldstein BJ, Aschner P, et al. Efficacy and safety of sitagliptin added to ongoing metformin and rosiglitazone combination therapy in a randomized placebo-controlled 54-week trial in patients with type 2 diabetes. J Diabetes. 2013;5:68-79.

13. Fonseca V, Staels B, Morgan JD, et al. Efficacy and safety of sitagliptin added to ongoing metformin and pioglitazone combination therapy in a randomized, placebo-controlled, 26-week trial in patients with type 2 diabetes. J Diabetes Complications. 2013;27:177-83.

14. Vilsboll T, Rosenstock J, Yki-Jarvinen H, et al. Efficacy and safety of sitagliptin when added to insulin therapy in patients with type 2 diabetes. Diabetes Obes Metab. 2010;12:167-77.

15. Mathieu C, Shankar RR, Lorber D, et al. A randomized clinical trial to evaluate the efficacy and safety of co-administration of sitagliptin with intensively titrated insulin glargine. Diabetes Ther. 2015;6:127-42.
16. Hermansen K, Kipnes M, Luo E, Fanurik D, Khatami $\mathrm{H}$, Stein P. Efficacy and safety of the dipeptidyl peptidase- 4 inhibitor, sitagliptin, in patients with type 2 diabetes mellitus inadequately controlled on glimepiride alone or on glimepiride and metformin. Diabetes Obes Metab. 2007;9:733-45.

17. Miettinen $\mathrm{O}$, Nurminen M. Comparative analysis of two rates. Stat Med. 1985;4:213-26.

18. Engel SS, Round E, Golm GT, Kaufman KD, Goldstein BJ. Safety and tolerability of sitagliptin in type 2 diabetes: pooled analysis of 25 clinical studies. Diabetes Ther. 2013;4:119-45.

19. Plosker GL. Sitagliptin: a review of its use in patients with type 2 diabetes mellitus. Drugs. 2014;74:223-42.

20. Zammitt NN, Frier BM. Hypoglycemia in type 2 diabetes: pathophysiology, frequency, and effects of different treatment modalities. Diabetes Care. 2005;28:2948-61.

21. Holstein A, Egberts EH. Risk of hypoglycaemia with oral antidiabetic agents in patients with type 2 diabetes. Exp Clin Endocrinol Diabetes. 2003;111:405-14.

22. Vexiau P, Mavros P, Krishnarajah G, Lyu R, Yin D. Hypoglycaemia in patients with type 2 diabetes treated with a combination of metformin and sulphonylurea therapy in France. Diabetes Obes Metab. 2008;10(Suppl 1):16-24.

23. Lopez JM, Annunziata K, Bailey RA, Rupnow MF, Morisky DE. Impact of hypoglycemia on patients with type 2 diabetes mellitus and their quality of life, work productivity, and medication adherence. Patient Prefer Adherence. 2014;8:683-92.

24. Davidson JA, Lajara R, Aguilar RB, Mattheus M, Woerle HJ, von Eynatten M. Efficacy and safety of linagliptin in Hispanic/Latino patients with type 2 diabetes mellitus: a pooled analysis from six randomized placebo-controlled phase 3 trials. BMJ Open Diabetes Res Care. 2014;2:e000020. 